\title{
Reflets
}

Revue ontaroise d'intervention sociale et communautaire

\section{Éducation juridique populaire sur les droits des femmes en Ontario}

\section{Andrée Côté}

Volume 3, numéro 2, automne 1997

Visibles et Partenaires : Pratiques et recherches féministes

URI : https://id.erudit.org/iderudit/026172ar

DOI : https://doi.org/10.7202/026172ar

Aller au sommaire du numéro

\section{Éditeur(s)}

Reflets : Revue ontaroise d'intervention sociale et communautaire

ISSN

1203-4576 (imprimé)

1712-8498 (numérique)

Découvrir la revue

Citer cet article

Côté, A. (1997). Éducation juridique populaire sur les droits des femmes en Ontario. Reflets, 3(2), 50-73. https://doi.org/10.7202/026172ar

\section{Résumé de l'article}

L'adoption de la Charte Canadienne des droits et libertés a suscité un regain d'intérêt pourl'éducation sur les droits de la personne. Par contre, la littérature semble avoir porté peud'attention à l'éducation juridique populaire sur les droits des femmes. L'auteure se proposeici de dégager certaines balises théoriques et méthodologiques pour un tel programme. Par lasuite, elle fait état d'un projet développé pour la Table féministe francophone de concertationprovinciale de l'Ontario intitulé Les Franco-Ontariennes et les droits à l'égalité, d'où aémergé un modèle de formation structuré en cinq temps : le développement et la rechercheaction(dont il sera peu question ici), la formation, la consultation des participantes, la concertationpolitique et la mise en oeuvre des recommandations. Il s'agit d'un modèle dynamique quiparticipe au mouvement progressif pour la réalisation effective des droits humains des femmes.Plus spécifiquement, ce modèle vise l'acquisition des connaissances juridiques de base,une compréhension de l'évolution historique, sociale et politique du droit, ainsi que l'opérationactuelle du «droit-en-action». Il vise aussi à dégager une définition par les participantes ellesmêmesdes principales embûches dans la réalisation progressive de leurs droits à l'égalité et laréalisation pratique du fait qu'elles sont toutes des agentes potentielles dans la transformationde l'ordre juridique dans lequel nous vivons.
Tous droits réservés (C Reflets : Revue ontaroise d'intervention sociale et communautaire, 1997
Ce document est protégé par la loi sur le droit d'auteur. L'utilisation des services d'Érudit (y compris la reproduction) est assujettie à sa politique d'utilisation que vous pouvez consulter en ligne.

https://apropos.erudit.org/fr/usagers/politique-dutilisation/ 


\section{Éducation juridique populaire sur les droits des femmes en Ontario}

L'adoption de la Charte Canadienne des droits et libertés a suscité un regain d'intérêt pour l'éducation sur les droits de la personne. Par contre, la littérature semble avoir porté peu d'attention à l'éducation juridique populaire sur les droits des femmes. L'auteure se propose ici de dégager certaines balises théoriques et méthodologiques pour un tel programme. Par la suite, elle fait état d'un projet développé pour la Table féministe francophone de concertation provinciale de l'Ontario intitulé Les Franco-Ontariennes et les droits à l'égalité, d'où a émergé un modèle de formation structuré en cinq temps: le développement et la rechercheaction (dont il sera peu question ici), la formation, la consultation des participantes, la concertation politique et la mise en oeuvre des recommandations. Il s'agit d'un modèle dynamique qui participe au mouvement progressif pour la réalisation effective des droits humains des femmes.

Plus spécifiquement, ce modèle vise l'acquisition des connaissances juridiques de base, une compréhension de l'évolution historique, sociale et politique du droit, ainsi que l'opération actuelle $d u$ "droit-en-action». Il vise aussi à dégager une définition par les participantes ellesmêmes des principales embûches dans la réalisation progressive de leurs droits à l'égalité et la réalisation pratique du fait qu'elles sont toutes des agentes potentielles dans la transformation de l'ordre juridique dans lequel nous vivons.

\section{Andrée Côté}

LL.B., LL.M., Toronto

\section{Introduction}

C'est grâce à Marie-Luce Garceau et à l'équipe organisatrice du colloque Visibles et partenaires, auquel j'ai été invitée à participer, 
"...développement

d'un modèle

d'éducation juridique

populaire articulé

autour de trois volets:

une formation

juridique sur les droits

à l'égalité, une

consultation au sujet

des politiques

gouvernementales

susceptibles de porter

atteinte aux droits à

l'égalité des fermmes et, enfin une rencontre

provinciale de

concertation sur les

stratégies pouvant être

mises en oeuvre..." que j'ai pu avoir l'occasion de réfléchir sur un aspect important de mon travail, à savoir l'éducation juridique populaire sur les droits des femmes. Cela fait maintenant vingt ans que je prépare et anime des sessions de formation à l'intention des groupes de femmes du Québec et de l'Ontario sur différents thèmes, notamment l'avortement, le droit de la famille, la discrimination, les droits des lesbiennes, le harcèlement sexuel, l'inceste, la violence conjugale, le fémicide ainsi que la problématique des droits à l'égalité et celle des droits humains des femmes. Mais c'est la première fois que je tente d'articuler mon projet éducatif et le texte qui suit n'est qu'une première étape dans une démarche à plus long terme.

Le 17 avril 1995 marquait le dixième anniversaire de l'entrée en vigueur des droits à l'égalité consacrés dans l'article 15 de la Charte canadienne des droits et des libertés. Le mouvement des femmes avait activement participé, au début des années 1980, à l'élaboration des termes de cet article, et son adoption avait suscité les plus grands espoirs (Eberts 1985). Dix ans plus tard, la Table féministe francophone de concertation provinciale de l'Ontario a jugé qu'il serait opportun de faire état de la jurisprudence développée à partir de cette disposition constitutionnelle et de mesurer son impact sur les droits à l'égalité des Franco-Ontariennes.

C'est à partir de cette idée que le projet, intitulé Les FrancoOntariennes et les droits à l'égalité garantis à l'article 15 de la Charte, a vu le jour. J'ai eu le mandat de concevoir et de diriger ce projet, avec l'aide d'un comité encadreur composé de représentantes de différents groupes venant de six régions de la province, membres de la Table féministe francophone de concertation provinciale de l'Ontario. Ce projet a donné lieu au développement d'un modèle d'éducation juridique populaire articulé autour de trois volets: une formation juridique sur les droits à l'égalité, une consultation au sujet des politiques gouvernementales susceptibles de porter atteinte aux droits à l'égalité des femmes et, enfin une rencontre provinciale de concertation sur les stratégies pouvant être mises en oeuvre afin de promouvoir la réalisation effective des droits à l'égalité.Avant de décrire plus en détail chacune des composantes 
de ce projet,j'aimerais toutefois faire état de certaines prémisses à partir desquelles nous avons travaillé.

\section{Éducation juridique populaire sur les droits des femmes : éléments de définition}

L'éducation en matière de droits de la personne est une chose relativement nouvelle (Seydegard 1984). De fait, une étude récente indique que parmi la population en général, c'est dans l'expérience personnelle et celle des membres de la famille ou des amis que les gens trouvent leurs principales sources d'information sur le droit et le système judiciaire (Ministère de la justice 1990:14). Ce système de transmission des connaissances juridiques connait bien des lacunes lesquelles se reflètent notamment dans le fait qu'une importante proportion des immigrantes et immigrants méconnaissent les lois et craignent le système judiciaire (Manley-Casimir, Cassidy et de Castell 1989). Toutefois, l'adoption de la Charte canadienne des droits et libertés a marqué un point tournant non seulement en ce qui regarde les assises de notre droit constitutionnel, mais aussi par rapport à l'importance que l'on accorde à l'éducation juridique des citoyens. Comme l'écrit l'équipe de Manley-Casimir, «more than at any other time in our history (...) there needs to be the conscious, intelligent and systematic cultivation of legal litteracy for the polulace as a whole» (1989: 87). D'ailleurs, il ne fait pas de doute que depuis l'adoption de la Charte, la démystification du droit gagne rapidement du terrain, comme le notent Ribordy, Laflamme et Cazabon (1986).

Aujourd'hui, de nombreux organismes gouvernementaux et non gouvernementaux ont intégré une composante «information juridique» dans leurs travaux: on en recensait pas moins de 862 en Ontario (Lane 1984). Mais même s'il existe une pléthore de documents informatifs et de guides pratiques portant sur des aspects spécifiques du droit, même si les établissements scolaires sont en voie d'intégrer un volet «droits de la personne» dans leurs 
«...il existe

relativement peu de programmes voués à l'éducation juridique populaire, et encore moins de programmes visant spécifiquement les femmes adultes qui oeuvrent pour la protection et la promotion des droits des femmes.» programmes, et même si quelques établissements universitaires ont introduit des programmes d'études spécifiques sur la question (Seydegard et Gibbs 1993), il existe relativement peu de programmes voués à l'éducation juridique populaire, et encore moins de programmes visant spécifiquement les femmes adultes qui oeuvrent pour la protection et la promotion des droits des femmes. Pourtant, on considère que l'éducation juridique est une des conditions essentielles à la promotion de la justice et des valeurs égalitaires et, ultimement, qu'elle est le gage d'une réelle démocratie (Webking 1989). Comme l'affirment Clarence Dias et David Gillies, "[legal] literacy is the key to exercising rights» (1993:13). Heureusement, depuis quelques années, plusieurs organisations non gouvernementales ont développé des manuels de formation juridique populaire qui sont fort intéressants (CEDPA 1995; Spector 1994; The Legal Ressources Foundation 1997).

Si j'utilise l'expression éducation juridique populaire, c'est pour marquer une certaine distance face aux approches fondées sur la notion d'information juridique. Par exemple, je pense à ce champ en expansion qui est le «public legal education and information» (mieux connu sous son acronyme PLEI) et sur l'idée que les personnes ont tout simplement besoin d'être informées du texte de loi afin d'être en mesure de défendre leurs droits. Cette approche est malheureusement celle la plus souvent utilisée dans les écoles, où l'on s'attend à ce que les étudiantes et les étudiants apprennent par coeur certaines règles sans comprendre le contexte dans lequel elles s'inscrivent, c'est aussi celle qu'on retrouve dans le matériel préparé à l'intention du grand public. Certains auteurs jugent très sévèrement cette méthode en affirmant qu'elle fournit une vision fragmentée et partielle du droit, sans jamais situer ses composantes comme un tout (Manley-Casimir, Cassidy et de Castell 1987: 83, 84). Ils soulignent de plus que ce type de connaissance juridique est rapidement périmé (Id. 1989: 92).

À mon avis, une approche qui se limite à résumer et à vulgariser le droit positif est non seulement réductrice (Graham et al.1990), mais elle risque de renforcer le statu quo et de servir d'outil de propagande au gouvernement. En effet, en l'absence d'une mise en contexte et d'une appréciation réaliste des droits énoncés dans 
la législation portant sur les droits de la personne, une telle approche peut servir à rehausser la crédibilité des gouvernements, alors même qu'ils continuent à mettre en oeuvre des politiques qui portent atteinte aux droits humains des femmes et des autres groupes historiquement victimes de discrimination. Comme l'écrivent Schuler et Kadirmagar-Rajasingham:

A legal literacy approach that merely gives women knowledge about laws, rights and obligations, or the functionning of the legal system in such a context, rests on shaky pedagogical ground. A danger inherent in legal literacy is that it can end up supporting the status quo. As a reflection of the spirit of the people, law reflects and reinforces not only the strenghts but the weaknesses of a society (Sobhan). Unless the strenghts and weaknesses are distinguished and confronted, legal literacy fulfills a retrogressive function (1995: 22-23).

«Pour avoir du droit un portrait réellement conforme au droit en vigueur, on doit également prendre en considération le «droit en action»..."
De plus, une présentation qui se limite à exposer des critères législatifs et réglementaires est rarement intelligible pour des nonjuristes. En effet, dans la mesure où tout le monde sait, ou tout le moins soupçonne, que l'opération du droit dépend de bien d'autres facteurs que les normes formellement établies dans les textes de lois, une présentation portant exclusivement sur le contenu des textes de lois n'a littéralement aucun sens. Ainsi faut-il garder à l'esprit que le "droit» ne se limite pas à la loi : il comprend non seulement la législation fédérale et provinciale, mais aussi la réglementation adoptée afin de mettre en oeuvre les textes législatifs, ainsi que la jurisprudence prononcée par les tribunaux et qui interprète les textes de loi. Pour avoir du droit un portrait réellement conforme au droit en vigueur, on doit également prendre en considération le "droit en action", c'est-à-dire le droit qui est élaboré dans le cadre de l'administration quotidienne de la justice et qui se caractérise tant par l'action que par l'inaction de l'État et de ses divers agents, ainsi que par celle de la magistrature (Rocher 1987). C'est le droit en action qui définit très concrètement les paramètres réels de l'ordre juridique dans lequel 
évoluent les différents groupes sociaux. Par ailleurs, le droit ne doit pas être présenté comme une autorité ahistorique et monolithique, comme c'est malheureusement souvent le cas. Il doit plutôt être situé dans son contexte politique, économique et social, appréhendé dans son évolution historique, comme un objet en mouvement qui répond aux diverses pressions émergeant du corps social (Eusebio 1989). Dans le développement de programmes d'éducation sur les droits des femmes, il est aussi impératif de situer le droit comme un des instruments historiquement utilisés par l'État pour assurer la subordination des femmes aux hommes (Dhavernas 1978; Boivin 1986; Pateman 1988; MacKinnon 1989; Smart 1989). Ainsi, doit-on rappeler que les branches législative, exécutive et judiciaire de l'appareil d'État d'origine européenne ont activement collaboré à l'élaboration d'un corpus juridique qui pose la domination patriarcale, le sexisme, le racisme et l'homophobie au coeur de ses codes civils et pénaux, ainsi que dans une foule de lois et de précédents judiciaires. Non seulement le droit a-t-il donné une base légale à la domination masculine, mais il est aussi important de souligner que les pratiques actuelles des hommes d'État et de la magistrature demeurent tributaires de ces règles maintenant formellement abolies (Brockman et Chunn 1993). De la même façon, on doit rappeler que le droit répond à des intérêts spécifiques de classe et de race, ayant été un instrument de répression de la classe ouvrière ainsi que de l'oppression des peuples autochtones et des minorités raciales. Faire abstraction de ces dimensions du droit établit à coup sûr une dissonance entre l'expérience individuelle et collective des femmes, et l'information juridique qui pourrait être offerte dans le cadre d'une session de formation. Du même coup, cela peut aussi amener les participantes à remettre en question la validité du contenu de la formation, et peut-être même la validité

«...pour éviter de sombrer dans la mythologie du droit, il est important de développer une analyse politique..." du discours sur les droits de la personne.

En d'autres termes, pour éviter de sombrer dans la mythologie du droit, il est important de développer une analyse politique du droit qui soit en mesure de l'objectifier, de le contextualiser et de le critiquer. Comme l'écrivent Schuler et KadirmagarRajasingham (1995:32) : "Only a political understanding of rights as 
fluid ressources and not as a finished product and a social fact, will overcome the shortcomings of the "myth" of rights". De plus, le droit doit être envisagé à partir d'une perspective tenant compte, par exemple, des intérêts collectifs des femmes, des peuples autochtones, des immigrantes et immigrants et des personnes à faible revenus. Une conception libérale des droits, qui met exclusivement l'accent sur les libertés individuelles (liberté d'expression, liberté de conviction politique) au détriment des droits collectifs, peut servir à camoufler les mécanismes de domination et les rapports de pouvoir. Comme l'écrit la professeure Sherene Razack (1993), l'approche individualiste des droits est très limitée :

Human rights are widely thought to be only about the preservation of the dignity and worth of the individual and equal opportunity (hence the popularity of the civil libertarian perspective) and not about connecting these to the high infant mortality rate among native populations, the poverty level of certain groups, systemic violence against women or even the issues of pay and promotion for women and minorities (...) discrimination is less a problem of prejudice and stereotyping and more a problem of power - power that enables the dominant group to oppress through a myriad of social, economic and political institutions as well as in countless episodes of individual behaviour (...) The individual rights model does not give us any conceptual tools for understanding oppression and the systems that constrain individual choice (p. 49).

L'éducation juridique populaire doit aussi tenir compte du fait que le droit est un terrain de lutte autour duquel les femmes se sont mobilisées afin d'obtenir des changements fondamentaux dans leurs conditions de vie. Le droit a évolué à tel point que la loi constitutionnelle du Canada reconnaît aujourd'hui que les droits à l'égalité des femmes représentent une des valeurs primordiales de la société canadienne. C'est bien grâce aux nombreuses générations de féministes et de personnes attachées aux droits humains qui ont contesté dans l'arène politique et 
"La critique féministe $d u$ droit s'est attaquée non seulement aux règles de fond, ou au droit substantif, mais également aux mécanismes de mise en oeuvre du droit et aux valeurs véhiculées par les membres de la profession juridique.» juridique le droit sexiste et patriarcal légué par les systèmes de common law et de droit civil napoléonien qu'il en est ainsi. La critique féministe du droit s'est attaquée non seulement aux règles de fond, ou au droit substantif, mais également aux mécanismes de mise en oeuvre du droit et aux valeurs véhiculées par les membres de la profession juridique. On pense notamment à l'immense travail accompli sur le droit et les pratiques judiciaires concernant la violence contre les femmes, particulièrement la violence conjugale et le viol. Mais il faut non seulement prendre acte des luttes qui ont été menées par les femmes pour transformer le droit, mais aussi tenir compte de la résistance interne des membres de la profession juridique à ces changements. Pensons, par exemple, à la question de la perquisition dans les dossiers personnels des victimes d'agression sexuelle, ce qui illustre la résistance actuelle de la profession juridique et de la magistrature aux amendements législatifs visant à restreindre les prérogatives sexuelles des hommes (Côté 1997). Ces attitudes et ces comportements influencent l'ordre juridique dans lequel les femmes évoluent tout autant que les textes de lois formant le droit positif.

Outre le fait que l'éducation juridique populaire doit permettre aux participantes d'acquérir certaines connaissances juridiques de base, être en mesure de bien faire comprendre le rôle du droit dans la société et développer une analyse critique face à ce rôle, elle doit, de surcroit, être capable de susciter chez les participantes une prise de conscience de leur position comme sujets de droit et de leur rôle potentiel dans le développement et la reconfiguration du droit. Ainsi, l'éducation juridique doit pouvoir fournir «an understanding of law in breath and depth, an understanding of law's role and function in society, the ability to think critically and the cultivaton of active citizen participation" (Manley-Casimir, Cassidy et de Castell 1989: 90).

Cette dernière caractéristique de l'éducation juridique, à savoir sa capacité de susciter une participation active des citoyennes à la transformation des rapports socio-juridiques, a été soulignée par divers auteurs. La professeure Sherene Razack plaide en faveur d'une «éducation radicale», susceptible de «restructurer les 
«D'autres soulignent

que l'éducation

juridique populaire

doit viser la prise en

charge par les

participantes et qu'elle

doit susciter une

pratique libératrice.» arrangements sociaux sur un mode plus équitable, juste et humanitaire» (Razack 1995: 44). D'autres soulignent que l'éducation juridique populaire doit viser la prise en charge par les participantes et qu'elle doit susciter une pratique libératrice (Graham et al. 1990). En effet, dans la mesure où le droit actuel demeure marqué dans sa substance et dans sa pratique par la jurisprudence de l'inégalité, la formation en vue d'une transformation égalitaire du droit est la condition sine qua non d'une éducation qui ne vise pas à renforcer le statu quo. Comme l'écrivent Schuler et Kadirmagar-Rajasingham (1995):

To the degree that women learn to «read» (i.e., understand, assert or critique and redefine) the cultural values and social norms embodied in the law, their authentic participation in the sociolegal and political process will be realized. Legal literacy can play an important role in bringing this about (...) Legal literacy must transcend the naive view that because constitutions give women the right to vote, the system is functional to women. It must overcome the mistaken notion that to participate, the only thing women need is information about their rights, and that if they assert their rights, they will have become full citizens. To be effective, legal litteracy's proponents must view social change and the transformation of social power relations as imperatives of the democratic process, and that women must be key players in deciding the changes to be made (p.36).

L'éducation juridique populaire doit donc concevoir le droit dans son évolution historique, dans son contexte social et politique, comme un instrument souvent manipulé par un groupe social en vue de promouvoir ses intérêts face à d'autres groupes, et qui fait l'objet de contestations judiciaires et de mobilisations politiques destinées à le transformer. 


\section{Description du projet «Les Franco-Ontariennes et les droits à l'égalité garantis à l'article 15 de la Charte canadienne des droits et libertés»}

C'est donc à partir de ces prémisses que nous avons développé un modèle d'éducation juridique populaire sur les droits à l'égalité des femmes destiné à la Table féministe francophone de concertation provinciale de l'Ontario. Ce projet a été conçu à l'intention de militantes oeuvrant dans des groupes de femmes en Ontario français, et donc de femmes possédant déjà une solide connaissance de différents aspects de la condition féminine. Les objectifs du projet consistaient à permettre aux participantes d'acquérir des connaissances de base relativement aux droits constitutionnels à l'égalité, de leur donner les outils pour procéder à une analyse critique des politiques gouvernementales à la lumière de ces garanties constitutionnelles, et de les aider à identifier les recours dont elles disposent pour intervenir en vue d'une transformation du droit.

Nous avons développé un modèle articulé en trois volets: une session de formation portant sur l'évolution historique et juridique des droits à l'égalité au Canada, une consultation destinée à identifier les politiques gouvernementales susceptibles de porter atteinte aux droits à l'égalité, et une rencontre de concertation provinciale sur les stratégies pouvant être mises en oeuvre afin de promouvoir la réalisation effective des droits à l'égalité. Ces trois volets ont été précédé par une phase de développement de projet et de recherche-action, et ont ensuite donné lieu à une phase de «suivi», au cours de laquelle nous nous sommes employées à mettre en oeuvre quelques-unes des recommandations issues du projet. En fait, il s'agit d'un projet en cinq étapes: développement et recherche-action, formation, consultation, concertation et suivi.

Un comité encadreur, composé de femmes oeuvrant dans différents groupes membres de la Table féministe ${ }^{1}$, a participé au développement du projet. Il a approuvé le contenu et la forme de 
la session de formation, ainsi que le questionnaire soumis lors des rencontres de consultation et l'ordre du jour de la rencontre provinciale de concertation. C'est également le comité encadreur qui a élaboré les critères de participation à ce projet, car un des objectifs était de veiller à ce que la diversité de la communauté francophone en Ontario soit convenablement représentée. La représentation équitable des participantes a d'ailleurs été soulignée dans d'autres programmes d'éducation juridique, comme celui développé par la professeure Razack pour le Centre de recherche et d'enseignement sur les droits de la personne de l'Université d'Ottawa (1993) ${ }^{2}$. Des coordonatrices locales ont transmis des suggestions au Comité encadreur en ce qui concerne le recrutement des participantes, et ces dernières ont pris en charge l'organisation matérielle des rencontres.

Les participantes étaient donc des femmes actives dans le mouvement des femmes, entendu dans son sens le plus large : des femmes qui militent contre les agressions sexuelles, la violence conjugale et l'abus sexuel des enfants; d'autres qui travaillent pour l'équité en emploi et l'équité salariale, pour la reconnaissance des droits des femmes handicapées, des droits des lesbiennes, des femmes immigrantes et des femmes de couleur, des femmes à faible revenu; ainsi que des femmes impliquées dans le secteur de la santé et des services sociaux, du mouvement syndical, de la défense des droits linguistiques et culturels et dans l'Église. La session de formation a été offerte dans cinq villes où l'on retrouve une forte concentration francophone, à savoir Sudbury,Timmins, Chatham, Toronto et Ottawa. De plus, nous avons invité quatre femmes de la région de Thunder Bay. Au total, 75 femmes ont participé à la session de formation. Nous avons eu confirmation de nos subventions au mois de septembre $1995^{3}$ et la première réunion du Comité encadreur a eu lieu au mois d'octobre 1995. La première session de formation a été offerte à Ottawa au mois de février 1996 et la rencontre provinciale de concertation a eu lieu au mois de mai suivant.

Nous avons retenu une approche participative à la formation, en tentant de faire profiter le groupe des connaissances, des compétences et de l'analyse critique que les militantes ont déjà 
acquises et développées au fil de leur expérience. Comme l'écrit la professeure Julie Macfarlane, «our personnal experience is the medium through which all our learning is filtered» (1994:374). C'est pourquoi une méthode qui tient compte de l'expérience des participantes est susceptible de transformer radicalement l'expérience d'apprentissage :

... in an experiential learning environment (...) the meaning of what it is to learn is transformed; the learner moves from being a spectator or a recipient of given knowledge to individual «meaning-making», through and from personal experience, supported by subsequent reflection. Experiential pedagogies draw direct relationships between learning and personal experiences. It is how we relate to and learn from our direct personal experiences in the active sense (rather than what we are told) which constitutes the most significant learning we do as adults (Macfarlane 1994: 375).

«...les participantes se sont livrées à une intense session de «remue méninges»; définissant la plupart du temps l'égalité par la négative et soulevant maints exemples de l'inégalité des femmes.»
La participation des femmes a ainsi été suscitée dès le début de la session de formation, lorsque nous leur avons demandé d'identifier les paramètres de «l'égalité des femmes»: pendant près d'une heure, les participantes se sont livrées à une intense session de «remue méninges»; définissant la plupart du temps l'égalité par la négative et soulevant maints exemples de l'inégalité des femmes. Cet exercice nous a permis de dégager le terrain à partir duquel nous allions travailler au cours des trois volets du projet et de susciter plusieurs questions et thèmes sur lesquels nous sommes revenues au cours de la session de formation afin de démarrer les consultations. Mais cet exercice a surtout permis de donner le ton au projet: c'est à partir de la voix des femmes et de leur expérience concrète de l'inégalité que notre réflexion s'est développée, et non pas à partir des catégories artificielles délimitées par le droit. Au fil de la formation, nous avons suscité d'autres sessions de «remue-méninges», à partir de questions portant, par exemple, sur les pratiques discriminatoires historiquement exercées par l'État ou sur les luttes menées par les femmes pour obtenir la reconnaissance de leurs droits. Nous avons aussi organisé une 
session de travail en petit groupe à partir d'un résumé de jurisprudence ${ }^{4}$, afin que les participantes puissent développer ellesmêmes une analyse critique face à l'approche formelle de l'égalité.

Par ailleurs, les participantes ont travaillé avec en main le texte de la Charte canadienne des droits et libertés, le Code des droits de la personne de l'Ontario, le Pacte international relatif aux droits économiques, sociaux et culturels et quelques autres textes législatifs. Tout en présentant les normes énoncées dans ces textes, nous demandions aux participantes de lire à voix haute différents articles et de les interpréter. Elles étaient ravies de constater que dans la plupart des cas, leur interprétation du texte juridique était juste. Comme l'a d'ailleurs souligné François-Xavier Ribordy et son équipe (1986), non seulement la population est-elle en mesure de comprendre la signification des textes juridiques originaux, mais il y a plusieurs avantages à les encourager à y avoir accès :

En vulgarisant un écrit déjà intelligible, on peut donner l'impression qu'il est originalement incompréhensible.

La vulgarisation peut devenir une nouvelle mystification, car elle peut tendre à tenir le citoyen à l'écart (...) Il reste que le droit n'est plus l'instrument spécialisé que seuls les avocats peuvent utiliser (1986: 26).

"Permettre à des nonjuristes d'avoir accès à des textes qui demeurent normalement la chasse gardée de la profession juridique est très "empowering»..."
Permettre à des non-juristes d'avoir accès à des textes qui demeurent normalement la chasse gardée de la profession juridique est très "empowering», si on me permet l'expression, car ces personnes se rendent compte qu'elles peuvent réellement comprendre le texte de loi (tout en étant conscientes que celui-ci sera aussi interprété par les tribunaux, et qu'elles doivent tenir compte de la jurisprudence). Ceci joue un rôle important dans la démystification du droit. Afin de nous assurer que les participantes puissent avoir un accès à ces textes dans le futur, nous avons préparé un cartable de documentation qui leur a été distribué gratuitement au début de la session de formation et qui contenait, outre les textes mentionnés plus haut, plusieurs autres lois provinciales et instruments internationaux, ainsi qu'une vingtaine d'articles et de textes de référence. Par ailleurs, au fur et à mesure que le projet progressait, nous y avons ajouté le rapport des rencontres 
de consultation (Côté 1996b), le rapport de la rencontre de concertation provinciale (Côté et Bouchard 1996) ainsi qu'un texte de référence sur l'évolution historique des droits constitutionnels à l'égalité (Côté 1996a). Ainsi, les participantes auront en main un document d'information et de référence qu'elles pourront consulter après la session de formation et ce pour se rafraîchir la mémoire ou pour approfondir certaines questions. Étant donné que l'information juridique devient rapidement périmée, le manuel de formation a été conçu pour permettre des mises à jour périodiques, et a été présenté dans un classeur à feuilles mobiles.

La session de formation durait une journée ${ }^{5}$ et abordait les thèmes suivants: la discrimination historiquement pratiquée par l'État, les luttes menées par les femmes contre ces politiques et la législation adoptée en réponse à leurs revendications; la distinction entre les différents régimes juridiques encadrant l'exercice des droits à l'égalité en Ontario (Code des droits de la personne, Loi canadienne sur les droits de la personne et Charte canadienne des droits et libertés); la définition, les domaines d'application et la portée des droits constitutionnels à l'égalité; la distinction entre l'égalité formelle et l'égalité substantive; la responsabilité de l'État dans la protection et la promotion des droits à l'égalité; la contestation judiciaire dans le contexte des causes-types menée depuis 1985

«Le second volet $d u$ projet consistait à mener une consultation auprès des femmes ayant participé à la session de formation, afin d'identifier les pratiques gouvernementales qui font obstacle à la concrétisation des droits à l'égalité garantis dans la Charte.» par les groupes de promotion des droits à l'égalité et le mandat du Programme de contestation judiciaire du Canada; les interventions féministes dans l'arène juridique et l'impact de leurs arguments sur le développement du droit; la jurisprudence développée par les tribunaux au sujet des droits à l'égalité consacrés par l'article 15 de la Charte et les recours disponibles en cas de violation des droits à l'égalité, en vertu de la Charte et de certains instruments internationaux.

Le second volet du projet consistait à mener une consultation auprès des femmes ayant participé à la session de formation, afin d'identifier les pratiques gouvernementales qui font obstacle à la concrétisation des droits à l'égalité garantis dans la Charte. Il s'agissait donc d'examiner la pratique législative, judiciaire, exécutive ou administrative des gouvernements fédéral et 
"Quelles sont les politiques des gouvernements provincial et fédéral qui portent atteinte aux droits à l'égalité des femmes?»

«...prévoir les justifications susceptibles d'être avancées par le gouvernement...» provincial sur la condition des femmes, et ainsi de déterminer dans quelle mesure l'État respecte et assure la promotion des droits constitutionnels à l'égalité. Les participantes étant toutes activement impliquées dans le mouvement des femmes, elles étaient particulièrement bien placées pour effectuer cet exercice. Les consultations ont été organisées dans les cinq villes où la session de formation avait été offerte et les femmes ayant participé à la session de formation étaient présentes. Nous avons élaboré un questionnaire pouvant être complété en trois ou quatre heures, avec des questions semi-structurées. Les questions portaient sur les politiques et les pratiques des gouvernement fédéral et provincial en matière de violence sexuelle, de violence conjugale, de séparation et divorce, de sécurité sociale, de droit au logement, de services de santé et de services sociaux, de droit au travail, de droit de l'immigration, etc. Dans chaque ville, les participantes ont choisi d'orienter leurs commentaires sur certains thèmes.

Les consultations commençaient par deux sessions de «remue méninges» en grand groupe portant sur les questions suivantes. Quelles sont les politiques des gouvernements provincial et fédéral qui portent atteinte aux droits à l'égalité des femmes? Ces politiques affectent-elles d'une façon particulière les femmes de différentes communautés, telles que les femmes francophones, autochtones, noires, handicapées, etc.? Par la suite, nous avons demandé à chaque groupe d'identifier quatre ou cinq exemples de politiques mentionnées qui soulèvent des enjeux importants pour les femmes et sur lesquels elles aimeraient réfléchir en profondeur. Le groupe a ensuite été divisé en équipes de travail, et chacune a approfondi les différents thèmes grâce à une série de questions sur les effets de la politique ou de la pratique gouvernementale sur la situation sociale, économique, culturelle, juridique ou politique des femmes, sur son impact sur les différentes communautés, et la menace qu'elle est susceptible de représenter pour certains droits garantis dans la Charte ou dans les instruments internationaux. Nous leur avons demandé de prévoir les justifications susceptibles d'être avancées par le gouvernement pour défendre sa politique et de les critiquer. Finalement, nous leur demandions d'identifier les solutions de rechange qu'il serait 
possible d'envisager pour promouvoir les droits à l'égalité des femmes sur ce plan. Chaque équipe de travail a ensuite présenté le résultat de son analyse au grand groupe. Les consultations se sont terminées par un exposé très sommaire des voies de recours disponibles (réforme législative, contestation judiciaire, forums internationaux et mobilisation populaire), et par l'identification de questions que les participantes souhaitaient approfondir et discuter lors de la rencontre de concertation provinciale du 26 mai 1996.

$\mathrm{Au}$ cours de ces consultations, les participantes ont donc identifié une multitude de politiques gouvernementales mettant en péril les droits à l'égalité des femmes, notamment les restrictions budgétaires dans les programmes contre la violence faite aux femmes; les restrictions dans l'aide juridique; l'introduction du "workfare»; les règles spéciales concernant l'immigration, notamment celles relatives au parrainage des femmes mariées; les coupures dans les programmes sociaux; l'abolition imminente du contrôle des loyers; les réformes en matière de droit du travail et l'abolition de la Loi sur l'équité en emploi; les coupures dans les services de garderies; les coupures dans les domaines de l'éducation et de la santé; l'insuffisance des services en français. Un rapport sur ces consultations a été rédigé quelques semaines après les rencontres et distribué à toutes les participantes pour qu'elles y ajoutent leurs commentaires. Ce rapport comprenait également une analyse juridique sommaire des problèmes identifiés par les participantes, un rappel des obligations constitutionnelles des gouvernements à l'égard des femmes, ainsi qu'une description des recours possibles. C'est sur la base de ce rapport que la rencontre provinciale de concertation a été organisée.

Le troisième volet de ce projet fut l'organisation d'une rencontre de concertation provinciale qui a eu lieu les 25 et 26 mai 1996 à Ottawa, afin d'analyser les questions soulevées dans le rapport des consultations et de discuter des diverses stratégies juridiques et politiques susceptibles d'être envisagées pour concrétiser les droits à l'égalité énoncés dans la Charte. Une trentaine de femmes des cinq villes où nous avions mené le projet ont participé à cet événement. Elles avaient toutes suivi la session 
"Trois priorités ont été retenues par les participantes, à savoir les droits des femmes immigrantes, la question $d u$ «workfare» et la violence contre les femmes.» de formation et participé aux consultations. La première journée fut consacrée à l'examen des politiques gouvernementales susceptibles de porter atteinte aux droits à l'égalité des FrancoOntariennes qui avaient été identifiées lors des consultations. Elle s'est terminée par une session de remue méninges très créative, portant sur différentes pistes d'action pour promouvoir l'égalité des femmes, y compris la création d'un parti féministe! La deuxième journée a donné lieu à des discussions sur les priorités et les stratégies possibles en vue de défendre les droits fondamentaux des femmes. Trois priorités ont été retenues par les participantes, à savoir les droits des femmes immigrantes, la question du «workfare» et la violence contre les femmes. Un rapport a été produit à la suite de cette rencontre et distribué à toutes les participantes.

\section{Bilan}

"Comme l'écrit une des femmes, " $j$ 'ai réalisé que je suis capable de lire des lois et de les comprendre..."
Le modèle qui a été développé à l'occasion de ce projet nous permet de tracer un bilan favorable, tant du point de vue de l'appréciation qu'en ont donné les participantes que des différentes idées et projets qui auront été engendrés par cette expérience.

Les participantes ont évalué le projet de manière très positive: elles disent avoir beaucoup appris, avoir eu de la facilité à comprendre la matière et les thèmes abordés et avoir envie d'approfondir leurs réflexions sur les droits des femmes. En ce qui concerne la session de formation comme telle, elles ont apprécié l'approche historique de l'évolution des droits et le fait de comprendre que cette évolution est le fruit de nos luttes. Elles ont beaucoup aimé faire le tour des différents textes de lois, prendre connaissance de la jurisprudence et comprendre le rôle des tribunaux dans l'interprétation du droit. Comme l'écrit une des femmes, «j'ai réalisé que je suis capable de lire des lois et de les comprendre. Cette journée a démystifié la loi pour moi, elle me pousse à ne plus me considérer victime, mais plutôt à augmenter mes connaissances». Finalement, les femmes ont aussi beaucoup 
apprécié l'approche participative, la diversité du groupe et le fait que l'animation peut être enrichie par les exemples et les commentaires de chacune. Elles ont souligné leur intention d'intégrer les connaissances acquises dans leurs analyses et stratégies et de tenter de les transmettre aux femmes avec qui elles travaillent.

Elles auraient cependant souhaité que la formation dure plus longtemps, afin de disposer de plus de temps pour assimiler la matière, recourir plus souvent au travail en petit groupe et explorer plus en détail certains thèmes et questions. Elles ont proposé plusieurs scénarios, allant d'une formation étalée sur plusieurs jours, à une formation en trois fins de semaines étalées au courant de l'année, en passant par des sessions hebdomadaires et la création d'un cours dans un collège communautaire. Elles aimeraient qu'une perspective internationale soit mieux intégrée à la présentation. Certaines auraient aimé recevoir le matériel pédagogique avant la formation. Elles souhaiteraient recevoir une mise à jour périodique de l'évolution des lois et de la jurisprudence, et voir développer certains dossiers spécifiques (comme par exemple l'équité en emploi, le droit de la famille, les droits des femmes immigrantes, l'avortement, etc.). Elles aimeraient assister à des rencontres locales ou provinciales sur les droits des femmes et participer à un réseau pour explorer comment utiliser leurs nouvelles connaissances dans des actions collectives concrètes. Elles soulignent qu'il serait intéressant d'avoir une trousse d'animation pour continuer à approfondir le dossier en petits groupes. Finalement, certaines souhaiteraient que l'on offre des cliniques juridiques populaires avec des avocates féministes à l'occasion desquelles les femmes pourraient obtenir de l'information sur des situations qui les concernent personnellement.

L'évaluation de l'ensemble du projet donne également lieu à un bilan extrêmement positif. On nous dit que ce projet avait été très motivant et qu'il avait suscité de l'enthousiasme chez des militantes qui sont parfois à bout de souffle. Les participantes ont jugé le rapport de consultation très facile à lire et ont ajouté que de voir leurs commentaires synthétisés et présentés par écrit leur faisait prendre conscience de la force qu'elles détiennent collectivement pour analyser l'impact des politiques gouvernementales 
"...ce projet s'est développé en véritable spirale, et il a débouché sur le développement d'autres projets qui, chacun à leur tour, risquent d'engendrer de nouvelles spirales de développement, en un mouvement exponentiel.» sur la situation des femmes. Les participantes sont d'avis que la rencontre de concertation provinciale a permis de consolider le «réseautage» entre femmes francophones de milieux très divers et de développer des moyens pour renforcer la solidarité des femmes en Ontario français.

Sur une note moins positive,je constate que ce projet a généré plus d'espoir et d'enthousiasme que nous étions en mesure d'en canaliser. En effet, nous n'étions pas préparées à donner suite aux projets qui ont été proposés par les participantes, ni à «récupérer» les militantes qui se sont portées volontaires pour développer les différents dossiers. Il est vrai qu'une telle tâche est ardue dans une province aussi vaste que l'Ontario, d'autant plus que la Table fonctionne entièrement à partir d'énergies bénévoles. En fait, nous n'avions même pas prévu organiser une réunion du Comité encadreur pour discuter des suites à donner au projet. Comme quoi il est difficile de sortir du moule classique du projet qui a un début et une fin, et de passer à une conception plus dynamique du travail d'éducation et de formation. En effet, ce projet s'est développé en véritable spirale, et il a débouché sur le développement d'autres projets qui, chacun à leur tour, risquent d'engendrer de nouvelles spirales de développement, en un mouvement exponentiel.

En ce qui concerne le suivi au projet, j'écrivais plus haut que trois priorités avaient été retenues par les participantes lors de la rencontre de concertation provinciale, à savoir les droits des femmes immigrantes, la question du «workfare» (en vertu duquel une personne est obligée d'effectuer un travail désigné afin de recevoir des prestations d'aide sociale) et la violence contre les femmes. Le rapport de la rencontre de concertation a été soumis à la Table féministe lors de sa rencontre du mois de décembre 1996, et une ébauche de projet de recherche a été soumise au Comité juridicopolitique de la Table féministe sur le thème Qui prend pays... l'impact du parrainage sur les droits à l'égalité des femmes immigrantes francophones. Le Comité a recommandé que la question des droits des immigrantes soit prise en charge à titre de dossier prioritaire pour l'année à venir. Un nouveau Comité encadreur pour ce projet a été mis sur pied, composé principalement de femmes 
immigrantes oeuvrant dans différents groupes membres de la Table féministe. Une première réunion de travail du Comité encadreur a eu lieu les 15 et 16 février 1997, et une demande de subvention a été rédigée et présentée au mois de mai.

D'une part, la Table féministe doit bientôt développer un projet sur la question du «workfare». Il s'agirait ici de recenser les différents textes analysant l'impact social, économique, politique et juridique $\mathrm{du}$ "workfare», en vue d'aider les groupes francophones qui travaillent sur cette question à développer des stratégies de résistance efficaces. En ce qui concerne la violence contre les femmes, c'est l'Action ontarienne contre la violence faite aux femmes qui a pris en main le suivi de ce dossier, notamment en donnant son appui à un rapport produit par la Ontario Association of Interval and Transition Houses (OAITH 1996) à l'intention de la Rapporteure spéciale sur la violence contre les femmes de l'ONU. Ce rapport portait sur l'impact des restrictions effectuées dans les dépenses publiques par les gouvernements fédéral et provincial sur les droits fondamentaux des femmes, notamment ceux qui sont énoncés dans la Déclaration contre la violence faite aux femmes (ONU 1994).

D'autre part, l'Action ontarienne a développé, en collaboration avec OAITH et METRAC, un projet de recherche sur l'impact des coupures récemment effectuées dans le Régime de l'aide juridique sur les femmes victimes de violence conjugale. Ce projet n'a malheureusement pas été financé. L'Action ontarienne a néanmoins soumis un bref mémoire portant sur cette question au Comité d'examen de l'aide juridique de l'Ontario au mois d'avril 1997 (AOcVF 1997). Dans son rapport, l'organisme fait notamment état des retombées des réformes apportées au régime de l'aide juridique sur les droits à l'égalité des femmes.

Par ailleurs, à la suite du projet sur les droits à l'égalité, la Table féministe est devenue membre du Programme de contestation judiciaire, lequel finance des causes-types sur les droits linguistiques et les droits à l'égalité. Après la dernière rencontre annuelle du Programme, en septembre 1996, une Coalition a été mise sur pied pour intervenir devant la Cour suprême du Canada dans l'affaire Rosenberg, sur la question du droit des conjointes lesbiennes et 
des conjoints gais de recevoir certains bénéfices sociaux dans le cadre d'une convention collective. Une membre de la Collective lesbienne y représentait la Table féministe. Finalement, la Table féministe a développé, en collaboration avec le Centre de recherches féministes, le Collège Glendon et le Collège des Grands Lacs, une proposition pour un cours d'éducation aux adultes. Ce cours viserait spécifiquement les femmes francophones et il porterait sur le thème très général de la condition des femmes en Ontario à l'aube de l'an 2000. Plusieurs des thèmes abordés lors du projet sur les droits à l'égalité seraient explorés pendant ce cours échelonné sur une période de 13 semaines. Bien qu'initialement offert à Toronto, ce cours pourrait éventuellement être enseigné dans différentes régions. Il reste à trouver des bailleurs de fonds pour ce projet.

\section{Conclusion}

«La méthode que nous avons empruntée est fondée sur la mise en valeur de l'expérience des participantes et elle vise à susciter chez elles une plus grande conscience de leur rôle dans l'évolution $d u$ droit et la transformation de l'ordre juridique.»
En conclusion, je crois que l'on peut dire que le modèle d'éducation juridique populaire sur les droits des femmes développé pour la Table féministe francophone de concertation provinciale de l'Ontario constitue un modèle intéressant. Il s'agit d'un projet qui s'articule autour de cinq étapes, à savoir le développement et la recherche-action, la formation, la consultation, la concertation et le suivi. Ce modèle génère un mouvement en spirale qui ouvre la voie à plusieurs autres sites d'intervention; à partir d'un travail sur les droits à l'égalité, le projet a donné naissance à trois nouveaux projets (immigration, "workfare» et violence contre les femmes) qui, à leur tour, déboucheront sans doute sur d'autres secteurs d'éducation juridique et d'intervention politique. La méthode que nous avons empruntée est fondée sur la mise en valeur de l'expérience des participantes et elle vise à susciter chez elles une plus grande conscience de leur rôle dans l'évolution du droit et la transformation de l'ordre juridique. À en juger par les indices préliminaires, il semble que ce modèle soit susceptible de générer 
l'enthousiasme des participantes et de leur permettre d'intégrer des connaissances juridiques relatives aux droits constitutionnels des femmes à l'égalité dans leur discours et dans leurs pratiques politiques.

\section{Bibliographie}

ACCESS RESEARCH ASSOCIATES (1986). A Survey of Canadian PLEI Providers, Ottawa, Section de la recherche et de la statistique, Statistique Canada.

AOCVF (1997). L'aide juridique et les femmes victimes de violence; présentation au Comité d'examen de l'aide juridique de l'Ontario, Ottawa, Action ontarienne contre la violence faite aux femmes.

BROCKMAN, Joan et Dorothy CHUNN (dir.) (1993). Investigating Gender Bias. Law, Courts and the Legal Profession, Toronto, Thompson Educational Publishing.

BOIVIN, Michelle (1986), «L'évolution des droits de la femme au Québec : un survol historique», Revue juridique la femme et le droit, vol. 2, no 1, 53-68.

CEDPA (1995). Training Trainers for Development, Conducting a Workshop on Participatory Training Techniques, Washington, The Centre for Development and Population Activities.

CÔTÉ,Andrée et Lyne BOUCHARD (1996). Rapport de la rencontre provinciale de concertation, Ottawa, Table féministe francophone de concertation provinciale de l'Ontario.

CÔTÉ, Andrée (1996a). Les droits constitutionnels à l'égalité : évolution historique et bilan actuel, Ottawa, Table féministe de concertation provinciale de l'Ontario.

CÔTÉ, Andrée (1996b). Rapport de consultation et identification des recours disponibles pour remédier à la violation des droits à l'égalité, Ottawa, Table féministe de concertation provinciale de l'Ontario.

CÔTÉ,Andrée (1997). Mémoire sur le projet de loi C-46 portant sur la communication des dossiers confidentiels dans les cas d'agression d'ordre sexuel, Ottawa,Action ontarienne contre la violence faite aux femmes.

DHAVERNAS, Odile (1978). Droits des femmes, pouvoir des hommes, Paris, Seuil.

DIAS, Clarence et David GILLIES (1993). Human Rights, Democracy and Development, Montréal, Centre international sur les droits de la personne et le développement démocratique.

EBERTS, Mary (1985). «Sex-Based Discrimination and the Charter», dans Anne Bayefsky et Mary Eberts (dir. de publ.), Equality Rights and the Charter of Rights and Freedoms, Toronto, Carswell, 183-218.

EUSEBIO, Dora (1989). «Reflexiones teoricas sobre el uso alternativo del Derecho», Portavoz Documentos (ILSA), vol. 3, 30-38.

GRAHAM, Marisa, Lina ARCIDIACONO, Valdenia BRITO et Macarena BRAVO (1990). «La Mujer y el Uso Alternativo del Derecho», Portavoz Documentos (ILSA), vol. 5, 46-55.

THE LEGAL RESSOURCES FOUNDATION (1997). Teaching Ourselves our Rights, A Manual for Community Trainers, vol. 2, Nairobi, Legal Ressources Foundation.

LANE, Marion (1984). Legal Education and Information in Ontario :A Preliminary Inquiry, Public, Toronto, Canadian Law Information Council.

MACKINNON, Catharine (1989). Towards a Feminist Theory of the State, Cambridge, Harvard University Press. 
MACFARLANE, Julie (1994). "A Feminist Perspective on Experience-Based Learning and Curriculum Change», Revue de droit d'Ottawa, vol. 26, no 2, 357-383.

MANLEY-CASIMIR, Michael, Wanda CASSIDY et Suzanne DE CASTELL (1987). Legal Literacy: Toward a Working Definition, Toronto, Canadian Law Information Council.

MANLEY-CASIMIR, Michael, Wanda CASSIDY et Suzanne DE CASTELL (1989). «The Charter of Rights and Freedoms and Legal Literacy» dans Keith McLeod (dir. de publ.) Canada and Citizenship Education, Toronto, Association canadienne d'éducation, 83-99.

MINISTÈRE DE LA JUSTICE (1990). Working Document: Focus Groups on Legal Education and Information Needs, document préparé pour le ministère de la Justice par Gallup Canada,Document WD 1991-1a.

OAITH (1996). Home Truth: Exposing the False Face of Equality and Security Rights for Abused Women in Canada. Submission to the UN Special Rapporteur on Violence Against Women, Toronto, OAITH.

PATEMAN, Carole (1988). The Sexual Contract, Stanford, Stanford University Press.

RAZACK, Sherene (1993). "Teaching Activists for Social Change: Coming to Grips with Questions of Subjectivity and Domination", Revue canadienne pour l'étude de l'éducation aux adultes, vol. 7, no 2, 43-56

RIBORDY, François-Xavier, Simon LAFLAMME et Benoît CAZABON (1986). L'éducation et l'information juridique: études exploratrices, Ottawa, ministère de la Justice du Canada.

ROCHER, Guy (1987), «La sociologie du droit au Québec: une nouvelle discipline en émergence», dans R. Bureau et P. Mackay (dir. de publ.), Le droit dans tous ses états: La question du droit au Québec (1970-87), Montréal, Wilson et Lafleur, 555-596.

SCHULER, Margaret et Sakuntala KADIRMAGAR-RAJASINGHAM (1995). «Legal Literacy:A Tool for Women's Empowerment» dans Margaret Schuler et Sakuntala Kadirmagar-Rajasingham (dir. de publ.), Legal Literacy: A Tool for Women's Empowerment, New-York, OEF int., 21-69

SEYDEGARD, Magda et Heather GIBBS (1993). Background Paper: Recent Developments in Human Rights Education in Canada and in the United States, Ottawa, Centre de recherche et d'enseignement sur les droits de la personne de l'Université d'Ottawa.

SEYDEGARD, Magda (1984). «Savoir pour agir :l'apprentissage des adultes et les droits de la personne», dans Marshall Conley (dir. de publ.), L'enseignement des droits de la personne, Wolfville, NouvelleÉcosse, The Acadia University Institute, 49-61.

SMART, Carol (1989). Feminism and the Power of Law, Londres, Routledge.

SPECTOR, Paul (1994) The Training of Trainers, Washington, The Institute for International Research.

WEBKING, Edwin (1989). «The Charter and the Teaching of Human Rights», dans Keith McLeod (dir. de publ.), Canada and Citizenship Education, Toronto, Association canadienne d'éducation, 73-82.

\section{Notes}

1. Ce comité était composé des organismes suivants: le Comité juridico-politique de la Table féministe francophone de concertation provinciale de l'Ontario, le Centre contre les agressions à caractère sexuel de Chaham-Kent, l'Action ontarienne contre la violence 
faite aux femmes, le Comité-réseau des intervenantes d'Ottawa, le Centre Victoria de Sudbury et l'Accueil francophone de Thunder Bay.

2. Dans ce programme, on avait adopté les critères suivants: un nombre égal d'hommes et de femmes, les deux tiers de personnes blanches et un tiers de personnes noires, cinq pour cent d'handicapées, cinq pour cent d'hommes gais ou de lesbiennes, et cinq pour cent d'Autochtones (Razack 1993: 46).

3. Ce projet a reçu l'appui financier de l'Office des affaires francophones, du Programme de contestation judiciaire du Canada, de la Direction générale de la condition féminine de l'Ontario, de Patrimoine Canada et de la Fondation Trillium.

4. Il s'agissait en l'occurence de l'arrêt Bliss c. P.G. Canada, [1979] 1 R.C.S. 183, où le juge Ritchie endossait les propos d'un des juges de la Cour d'appel fédérale à l'effet que «si l'article 36 [de la Loi sur l'assurance-chômage] ne traite pas les femmes enceintes au chômage comme d'autres chômeurs, hommes ou femmes, c'est à mon sens parce qu'elles sont enceintes, et non parce qu'elles sont des femmes» (p. 190-191).

5. Ce qui s'est avèré être trop court. Plutôt que de bousculer les participantes, nous avons réaménagé l'horaire afin qu'elle dure une journée et demie. 